\title{
Indicaciones de cirugía antirreflujo: $¿$ Buenos y malos candidatos para la cirugía? Revisión bibliográfica y experiencia personal
}

\author{
ITALO BRAGHETTO M., OWEN KORN B., HÉCTOR VALLADARES H., \\ JOHANNA SILVA C. VERÓNICA AZABACHE C.
}

\section{Indications for antireflux surgery. A clinical experience and review of the literature}

\begin{abstract}
Antireflux laparoscopic surgery has excellent results in terms of improvement of symptoms, esophagitis, gastroesophageal sphincter competence and abnormal acid reflux. Indications for surgery are well established, however some of these are controversial. This is a review of the present indications for surgery in gastroesophageal reflux. The surgical indication should be the result of a complex clinical and laboratory work up. Patients with a clear cut surgical indication should be differentiated from those with doubtful indications, that require further analysis and those that are bad candidates for surgery. Young patients with macroscopic esophagitis, an incompetent sphincter, abnormal acid reflux test, that have a partial or negative response to treatment with proton pump inhibitors are those with the best surgical results. Bad candidates are patients with a psychiatric background, with atypical symptoms and those with a normal acid reflux test. In our experience with 935 patients, only 23\% had a surgical indication.
\end{abstract}

(Rev Med Chile 2010; 138: 605-611).

Key words: Esophagitis; Gastroesophageal reflux; General Surgery.

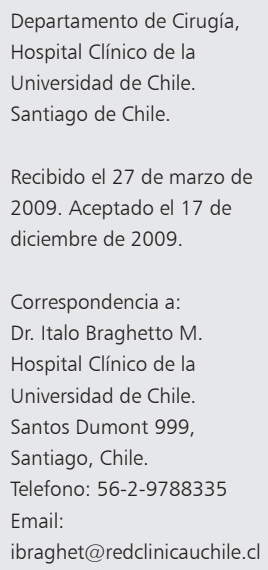

E n este artículo se realizó una revisión bibliográfica de trabajos publicados en relación a los factores de los pacientes que influyen en la indicación quirúrgica de la enfermedad por reflujo gastroesofágico (ERGE), con el fin de conocer e integrar las posturas existentes para asegurar buenos resultados post operatorios. Se revisó la bibliografía en la base de datos PubMed, utilizando los términos "surgical treatment" AND "gastroesophageal reflux", lo que arrojó un total de 7.826 artículos, de los cuales 1.431 eran catalogados como "review". De ellos se escogieron 20 artículos que de acuerdo a los autores fueron considerados los más atingentes al tema de la controversia en el tratamiento. Además se expone nuestra experiencia recopilada en los últimos 12 años en el Hospital Clínico de la Universidad de Chile.
Tanto el tratamiento médico como quirúrgico de la ERGE tienen como objetivos lograr la mejoría de los síntomas de la enfermedad, los mecanismos antirreflujo alterados y, por consiguiente, la calidad de vida del paciente. Ambos tratamientos son capaces de alcanzar tales resultados. Sin embargo, el tratamiento médico tiene la desventaja de que debe aplicarse a permanencia, presenta una recurrencia de $82 \%$ a los 6 meses de suspendido y la persistencia o progresión de la enfermedad es de $75 \%$ a los 10 años ${ }^{1}$ (Nivel de evidencia $2 c$, Recomendación nivel B). En contraparte, existen estudios que evidencian que se pueden lograr resultados más efectivos y permanentes con la aplicación del tratamiento quirúrgico, con excelente control de los síntomas a lo largo del seguimiento postoperatorio, tanto en las experiencia extranje- 
ras como en nuestra propia experiencia ${ }^{2,3}$ (Nivel de evidencia 1b, 2a, Recomendación A,B) (Tabla 1).

Las indicaciones de tratamiento quirúrgico para pacientes con ERGE resultan de un análisis objetivo del paciente, el cual considera: la historia clínica especificando los síntomas, los tratamientos previos realizados, las características del paciente, las enfermedades asociadas; el análisis del daño esofágico con endoscopia y biopsia, en la que encontramos enfermedad sin esofagitis erosiva (NERD) (60\%), esofagitis (40\%), y en 10\% de los pacientes se observan complicaciones derivados de un esófago de Barrett. Por otra parte, debe efectuarse el análisis de la función motora del cuerpo esofágico y del esfínter gastroesofágico (EGE) con manometría y, por último, el test de reflujo ácido y estudio de reflujo biliar determinado por el monitoreo de $\mathrm{pH}$ de $24 \mathrm{~h}$ y por el test de Bilitec que evalúan la calidad y severidad del material que refluye, el cual se realiza sólo en los casos correspondientes.

Algunos expertos ${ }^{3}$ recomiendan el tratamiento quirúrgico para la cirugía antirreflujo en los siguientes casos:

- Pacientes que no responden a terapia médica.

- Pacientes que responden bien pero recaen precozmente al auto suspender el tratamiento médico.

- Pacientes que requieren aumento de su dosis o evidencian progresión de la enfermedad (40\% a largo plazo).

- Pacientes jóvenes con ERGE crónico que necesitan terapia médica a permanencia (costo y duración).

- Pacientes que no están dispuestos a terapia médica (costo, otras razones).

- Complicaciones como esófago de Barrett, úlcera, estenosis, enfermedad respiratoria, esofagitis grado I-II con esfínter gastroesofágico incompetente y test de reflujo $(+)$, hernia hiatal verdadera.

- Síntomas extraesofágicos secundarios a ERGE.

\section{Consideraciones para cirugía antirreflujo. ¿Buenos o malos candidatos?}

Podríamos dividir a los pacientes en 3 categorías: los buenos candidatos, candidatos cuya indicación es controversial y los malos candidatos.

Buenos candidatos: Con nivel de evidencia $2 \mathrm{a}$ y 2 b y nivel de recomendación $B$, se han reportado

\section{Tabla 1. Síntomas de reflujo gastroesofágico antes del tratamiento quirúrgico y a los 12 meses postoperatorio}

\begin{tabular}{|c|c|c|}
\hline Síntomas & $\begin{array}{l}\text { Postoperatorio } \\
\text { Literatura } \\
\text { n }=5.326\end{array}$ & $\begin{array}{l}12 \text { meses de } \\
\text { seguimiento } \\
\text { Nuestra } \\
\text { experiencia } \\
n=219\end{array}$ \\
\hline Pirosis & 319 (6\%) & $23(10,5 \%)$ \\
\hline $\begin{array}{l}\text { Dolor } \\
\text { epigástrico }\end{array}$ & $107 \quad(2 \%)$ & $7 \quad(3,2 \%)$ \\
\hline $\begin{array}{l}\text { Regurgi- } \\
\text { tación }\end{array}$ & $107 \quad(2 \%)$ & $9 \quad(4,1 \%)$ \\
\hline Disfagia & $160 \quad(3 \%)$ & $11(5,0 \%)$ \\
\hline $\begin{array}{l}\text { Síntomas } \\
\text { respiratorios }\end{array}$ & 319 (2\%) & $21(9,5 \%)$ \\
\hline $\begin{array}{l}\text { Globus } \\
\text { faringeo }\end{array}$ & $160 \quad(3 \%)$ & - \\
\hline Flatulencia & 959 (18\%) & - \\
\hline Vómito & 0 & 0 \\
\hline Hemorragia & 0 & 0 \\
\hline
\end{tabular}

algunos factores que predicen un éxito quirúrgico en estos pacientes y son principalmente ${ }^{4,5}$ :

- pH metría de 24 h anormal.

- Síntomas típicos.

- Respuesta a terapia de supresión ácida.

- Índice masa corporal (IMC) $<35$.

- Jóvenes.

- RGE con esofagitis, esófago de Barrett corto.

- La presión de reposo del EGE en pacientes con reflujo ácido positivo y síntomas típicos no afectan el resultado exitoso.

En la Tabla 2 se muestran los factores que se correlacionan con mayor éxito postoperatorio.

Candidatos controversiales: Estos candidatos son los más complejos, por la incertidumbre en los resultados del tratamiento quirúrgico. Existen algunas características que deben ser evaluadas y que nos permitirán recomendar la cirugía.

a) Respuesta a inhibidores de la bomba de protones (IBP). Los resultados de la literatura no apoyan la hipótesis de que una mala respuesta a los IBP equivale a un pobre resultado después de la cirugía $^{6}$ (Nivel de evidencia $2 c$ ). Por consiguiente, aquel paciente refractario a la terapia médica no 
Tabla 2. Asociación univariada como factores de buen pronóstico en pacientes clasificados como buenos candidatos a cirugía

\begin{tabular}{|lccc|}
\hline $\begin{array}{l}\text { Variables a } \\
\text { considerar }\end{array}$ & $\begin{array}{c}\text { Excelente/ } \\
\text { bueno } \\
\%\end{array}$ & Malos & p \\
\hline $\begin{array}{l}\text { Respuesta a la terapia } \\
\text { de supresión de ácido } \\
\text { Completo/parcial }\end{array}$ & 92 & & \\
Menor/ninguno & 68 & 32 & 0,00008 \\
Síntomas & & 8 & \\
Típicos & 92 & 32,5 & 0,0001 \\
Atípicos & 67,5 & & \\
$\begin{array}{l}\text { Resultado del pH } \\
\text { de esófago 24 h }\end{array}$ & & 9 & \\
Anormal & 91 & 34,5 & 0,001 \\
Normal & 65,5 & & \\
\% Tiempo de pH \\
de esófago < 4
\end{tabular}

debe ser referido a cirugía sin un muy buen análisis previo y discusión del caso ${ }^{6}$. De la revisión de la literatura se desprende que aquellos pacientes que mejor responden a la terapia médica son los que tendrían mejores resultados postoperatorios. Sin embargo, hemos atendido pacientes que no tienen una buena respuesta o presentan respuesta parcial a los inhibidores de la bomba de protones y que en su evaluación preoperatoria se demuestra el esfínter incompetente o test de reflujo muy anormal o con manifestaciones de reflujo extraesofágicas, los que después de la cirugía resultan muy satisfechos con los resultados, con mejoría significativa de sus síntomas.

b) Tipo de reflujo. Algunos estudios sugieren que pacientes con reflujo de pie tienen peores resultados y otros, sin embargo, evidencian que la categorización de pacientes según el patrón de reflujo no tiene valor para predecir resultados ni en la selección de pacientes para la cirugía.

c) Síntomas respiratorios. Existen pacientes que sufren de síntomas faríngeos, laríngeos o respiratorios (tos irritativa, carraspeo matinal, disfonía, quiebre tonal o voz bitonal especialmente matinal, asma, etc) con pruebas funcionales contradictorias; generalmente no presentan esofagitis endoscópica pero si los síntomas persisten deben se sometidos a estudios para determinar reflujo (monitoreo de $\mathrm{pH}$ de $24 \mathrm{~h}$, Bilitec o impedanciometría) y, de confirmarse la presencia de reflujo, deben ser operados (Figura 1). Otros aluden causas multifactoriales de los síntomas laríngeos y, de ser así, no hay predictores de éxito postoperatorio para estos pacientes. Por todo ello es indispensable una evaluación completa del cuadro clínico y de laboratorio antes de decidir una cirugía y de este modo asegurar su éxito y poder reportar excelentes resultados después de cirugía en relación a la mejoría o disminución de los síntomas laríngeos o respiratorios ${ }^{7-9}$.

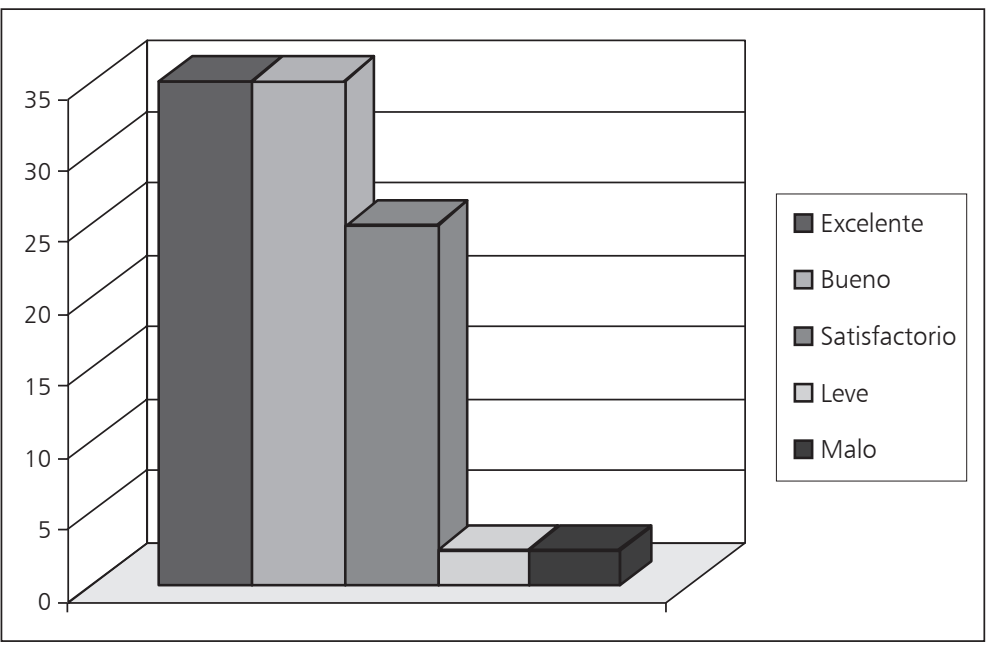

Figura 1. Evaluación de los resultados quirúrgicos de pacientes con síntomas respiratorios. 


\section{¿Qué candidatos tienen mayor probabilidad de falla dentro de este grupo?}

Los pacientes con síntomas atípicos, obesidad mórbida, o aquellos que no responden a la administración de medicamentos, deben ser informados de su mayor riesgo de fracaso quirúrgico. Sin embargo, algunos pacientes en estos grupos tienen éxito, por lo que es necesario realizar mayor investigación para analizar el beneficio real de la cirugía en ellos ${ }^{4}$ (Nivel de evidencia $2 b$, Recomendación B). En la Tabla 3 se muestran los factores asociados a la mayor o menor probabilidad de éxito en la cirugía.

Enfermedad no erosiva por reflujo (NERD). Estudios sugieren que el tratamiento quirúrgico es una valiosa opción de tratamiento para los pacientes con ERGE y síntomas persistentes, independientemente del aspecto endoscópico de la mucosa esofágica ${ }^{10}$. Otros exponen que la cirugía es una excelente opción de tratamiento para pacientes bien seleccionados con ERGE y persistencia de síntomas relacionados que no tienen evidencia endoscópica de esofagitis ${ }^{11}$ (Nivel de evidencia 3a). No obstante, otros resultados parecen más desalentadores en pacientes con NERD especialmente en pacientes de sexo femenino, en los que se describe que casi un tercio de ellos deben continuar con tratamiento médico $y$, por consiguiente, se concluye que la cirugía no presenta ventajas en cuanto a efectividad, costo y riesgo de complicaciones ${ }^{12}$ (Nivel de evidencia 3a, Recomendación grado B). Estos resultados ponen de manifiesto la necesidad de efectuar un exhaustivo análisis del estudio preoperatorio y de una cuidadosa selección de los pacientes antes de la cirugía antirreflujo. Se estudió en una encuesta a pacientes respecto de su calidad de vida (Puntaje GIQLI) antes y después de cirugía antirreflujo en un grupo de pacientes con o sin esofagitis erosiva en comparación con controles sanos y los resultados fueron muy similares en los 3 grupos, demostrando los buenos resultados de la cirugía tanto en pacientes con esofagitis erosiva como en aquellos pacientes con enfermedad por reflujo sin daño en la mucosa esofágica ${ }^{14}$.

Trastornos motores asociados. Los trastornos motores dependen de la severidad, y no constituyen una contraindicación para la fundoplicatura.
Tabla 3. Tasa de éxito de la cirugía acuerdo a diferentes variables

\begin{tabular}{|c|c|c|c|}
\hline Variable & $\begin{array}{l}\text { Tasa de } \\
\text { éxito }\end{array}$ & $\begin{array}{l}\text { Valor } \\
\text { de } p\end{array}$ & OR \\
\hline Hombre & 79 & 0,19 & 1,59 \\
\hline Mujer & 71 & & \\
\hline$<50$ Años & 76 & 0,8 & 1,09 \\
\hline$>=50$ Años & 74 & & \\
\hline \multicolumn{4}{|l|}{ IMC } \\
\hline$<35$ & 78 & 0,0036 & 4,81 \\
\hline$>35$ & 43 & & \\
\hline \multicolumn{4}{|l|}{ Respuesta a IBP } \\
\hline Sí & 77 & 0,035 & 2,64 \\
\hline No & 56 & & \\
\hline \multicolumn{4}{|c|}{ Patología psiquiátrica } \\
\hline Sí & 76 & 0,064 & 2,53 \\
\hline No & 55 & & \\
\hline Síntomas típicos & 85 & $<0,01$ & 7,75 \\
\hline Síntomas atípicos & 41 & & \\
\hline \multicolumn{4}{|c|}{ Cirugía abdominal previa } \\
\hline Sí & 72 & 0,83 & 1,09 \\
\hline No & 70 & & \\
\hline Sin hernia hiatal & 77 & 0,87 & 1,12 \\
\hline Hernia hiatal & 75 & & \\
\hline Sin esofagitis & 72 & 0,46 & 0,67 \\
\hline Esofagitis & 79 & & \\
\hline $\begin{array}{l}\text { Sin esófago de } \\
\text { Barrett }\end{array}$ & 65 & 0,27 & 0,47 \\
\hline $\begin{array}{l}\text { Con esófago de } \\
\text { Barrett }\end{array}$ & 80 & & \\
\hline
\end{tabular}

Por lo tanto, la cirugía antirreflujo es segura y efectiva como tratamiento de ERGE garantizando el mejoramiento de los síntomas de reflujo y una efectiva fundoplicatura mejora también la motilidad del esófago en muchos de estos enfermos. ${ }^{13,14}$ Otros autores han sugerido una fundoplicatura parcial (técnica de Toupet) para estos pacientes con disfunción motora, pero se ha demostrado que la fundoplicatura total, aparte de mejorar la presión del esfínter gastroesofágico, reestablece la peristalsis normal en la mayoría de los pacientes ${ }^{15,16}$. 
Colon irritable. El síndrome de intestino irritable no tiene un efecto negativo sobre los resultados de la cirugía antirreflujo por vía laparoscópica. La corrección quirúrgica de la ERGE puede mejorar la severidad de los síntomas de colon irritable ${ }^{17}$.

Vaciamiento gástrico. Se describe en algunos estudios que $40 \%$ de los pacientes con ERGE tienen retardo en el vaciamiento gástrico y sugieren que no existe relación entre el patrón de vaciamiento gástrico y la falla postoperatoria ${ }^{6}$.

Esófago de Barrett. Pocos autores discuten la indicación quirúrgica en Barrett extenso o complicado. Está bien documentado que la terapia médica no tiene buenos resultados a largo plazo, el gran problema es que la fundoplicatura de Nissen tiene una alta tasa de recurrencia en ellos, por lo tanto la controversia se genera frente a la elección de la técnica quirúrgica y la selección adecuada de pacientes. Sin embargo, estudios recientes muestran que la cirugía antirreflujo puede lograr una regresión de la metaplasia intestinal en pacientes con esófago de Barrett y puede, por lo tanto, alterar la historia natural de la enfermedad ${ }^{18,19}$ (Nivel de evidencia 2a, 2c, Grado de Recomendación B) (Tabla 4).

Malos candidatos. Los pacientes con ERGE y depresión mayor deben ser seleccionados cuidadosamente, incluso si son buenos candidatos para la cirugía desde un punto de vista fisiológico. En estos pacientes la cirugía puede normalizar las alteraciones, pero algunos han demostrado menos alivio sintomático, disfagia postoperatoria y escasa mejoría de calidad de vida. La funduplicatura de Toupet por vía laparoscópica que se utiliza con es- tos pacientes puede dar como resultado un mejor resultado subjetivo ${ }^{20}$.

El nivel de somatización en un paciente, puede ser útil para predecir los pobres resultados después de la cirugía antirreflujo. El conocimiento de los trastornos psicológicos puede mejorar la selección de un tratamiento óptimo para la ERGE y ayudar a reducir la tasa actual de los síntomas post quirúrgicos.

No es aconsejable operar cuando:

- Los pacientes no responden a IBP sin una cuidadosa y completa evaluación objetiva del cuadro clínico y los exámenes de laboratorio.

- Síntomas atípicos sin un estudio de la función esofágica.

- Vomitadores.

- Test de reflujo ácido normal (pH 24 h).

- Trastorno motor severo. Puede que tenga síntomas post operatorios no asociados a ERGE.

La experiencia en nuestro centro hospitalario en los últimos 12 años, evidencia algunos aportes interesantes (Tabla 5):

De un total de 935 pacientes estudiados por síntomas de reflujo gastroesofágico, en 219 (23,4\%) se resolvió quirúrgicamente. De los pacientes con reflujo gastroesofágico con síntomas típicos ( $\mathrm{n}=$ $640)$, se trataron con cirugía $73(11,4 \%)$. De aquellos que consultaron por síntomas laríngeos (106), sólo $12(11,3 \%)$ se operaron. De los pacientes que tenían asociado un esófago de Barrett $(n=55)$, se operaron 53 (96,3\%). De los que tenían hernia hiatal, se operó el 100\%. La Tabla 5 muestra los resultados del análisis de la indicación quirúrgica del reflujo gastroesofágico en el Hospital Clínico de la Universidad de Chile, desde 1995.

Tabla 4. Regresión Barrett corto versus extenso post cirugía antirreflujo

\begin{tabular}{|lcccc|}
\hline & \multicolumn{3}{c|}{ Regresión } \\
Primer autor & Año & SSBE & LSBE & p \\
Oelschlager & 2003 & $30 / 54(55 \%)$ & $0 / 36(0 \%)$ & $<0,001$ \\
Gurski & 2003 & $11 / 32(34 \%)$ & $0 / 21(0 \%)$ & $<001$ \\
Zaninotto & 2005 & $6 / 11(54 \%)$ & $0 / 24$ & $<0,001$ \\
Biertho & 2007 & $23 / 59(39 \%)$ & $0 / 11$ & $<0,001$ \\
Csendes & 2007 & $20 / 31(64 \%)$ & $26 / 42(62 \%)$ & \\
\hline
\end{tabular}

SSBE: Barrett Corto. LSBE: Barrett Largo. Referencias 18: Zaninotto G. Curr Opin Gastroenterol 2007; 23: 452. 19: Csendes A. Surgery 2006; 139: 46-53. 
Indicaciones de cirugía antirreflujo. Revisión bibliográfica y experiencia personal - I. Braghetto M. et al

Tabla 5. Indicación quirúrgica en pacientes con enfermedad por reflujo gastroesofágico Hospital Clínico, Universidad de Chile. (1995-2008)

\begin{tabular}{|lccc|}
\hline Diagnóstico & n de pacientes estudiados & n de pacientes operados & Porcentaje (\%) \\
\hline RGE & 640 & 73 & 11,4 \\
RGE + Laringitis & 106 & 12 & 11,3 \\
RGE + Barrett & 55 & 53 & 96,3 \\
RGE + Hernia hiatal & 81 & 81 & 100 \\
Otros & 53 & 219 & 0 \\
Total & 935 & & 23 \\
\hline
\end{tabular}

RGE: Reflujo Gastroesofágico.

\section{Conclusión}

De acuerdo a la revisión realizada, podemos concluir que para asegurar buenos resultados post operatorios los pacientes deben ser evaluados adecuadamente desde un punto de vista clínico, de la función esófago gástrica, identificar patología asociada y enfermedades siquiátricas.

La correcta selección de pacientes y una técnica quirúrgica adecuada nos permite tener buenos resultados.

\section{Referencias}

1. Ciovica R, Gadenstätter M, Klingler A, Lechner W, Riedl O, Schwab GP. Quality of life in GERD patients: medical treatment versus antireflux surgery. J Gastrointest Surg 2006; 10: 934-9.

2. Lundell L, Miettinen P, Myrvold HE, Hatlebakk JG, Wallin L, Malm A, et al. Nordic GORD study group: Sevenyear follow-up of a randomized clinical trial comparing proton-pump inhibition with surgical therapy for reflux oesophagitis. Br J Surg 2007; 94: 198-203.

3. Hinder RA, Libbey JS, Gorecki P, Bammer T. Antireflux surgery. Indications, preoperative evaluation and outcome. Gastroenterol Clin NA 1999; 28: 987-1005.

4. Morgenthal CB, Lin E, Shane MD, Hunter JG, Smith CD. Who will fail laparoscopic Nissen fundoplication? Preoperative prediction of long-term outcomes. Surg Endosc 2007; 21: 1978-84.

5. Campos GM, Peters JH, DeMeester TR, Oberg S, Crookes PF, Tan S, et al. Multivariate analysis of factors predicting outcome after laparoscopic Nissen fundoplication. J Gastrointest Surg 1999; 3: 292-300.

6. Wilkerson PM, Stratford J, Jones L, Sohanpal J, Booth
MI, Dehn TC. A poor response to proton pump inhibition is not a contraindication for laparoscopic antireflux surgery for gastro esophageal reflux disease. Surg Endosc 2005; 19: 1272-7.

7. Ciovica R, Gadenstätter M, Klingler A, Neumayer C, Schwab GP. Laparoscopic antireflux surgery provides excellent results and quality of life in gastroesophageal reflux disease patients with respiratory symptoms. J Gastrointest Surg 2005; 9: 633-7.

8. Salminen P, Sala E, Koskenvuo J, Karvonen J, Ovaska J. Reflux laryngitis: a feasible indication for laparoscopic antireflux surgery? Surg Laparosc Endosc Percutan Tech 2007; 17: 73-8.

9. Christian DJ, Buyske J. Current status of antireflux surgery. Surg Clin North Am 2005; 85: 931-47.

10. Bammer T, Freeman M, Shahriari A, Hinder RA, DeVault KR, Achem SR. Outcome of laparoscopic antireflux surgery in patients with nonerosive reflux disease. J Gastrointest Surg 2002; 6: 730-7.

11. Kamolz T, Granderath FA, Schweiger UM, Pointner R. Laparoscopic Nissen fundoplication in patients with nonerosive reflux disease. Long-term quality-of-life assessment and surgical outcome. Surg Endosc 2005; 19: 494-500.

12. Thibault R, Coron E, Sébille V, Sacher-Huvelin S, Bruley des Varannes S, Gournay J, Galmiche JP. Antireflux surgery for non-erosive and erosive reflux disease in community practice. Aliment Pharmacol Ther 2006; 24 : 621-32.

13. Pizza F, Rosetti G, Del Genio G, Maffettone V, Brusciano L, Del Genio A. Influence of esophageal motility on the outcome of laparoscopic total fundoplication. Dis Esophagus 2008; 21: 78-8.

14. Novitsky YW, Wong J, Kercher KW, Litwin DE, Swantrom LL, Heniford BT. Severely disordered esophageal peristalsis is not a contraindication to laparoscopic. 
Nissen fundoplication Surg Endosc 2007; 21: 950-4.

15. Herbella FAM, Tedesco P, Nipomnick I, Fisichella PM, Patti M. Effect of partial and total laparoscopic fundoplication on esophageal body motility. Surg Endosc 2007; 21: 285-8.

16. Heider TR, Farrell TM, Kircher AP, Colliver CC, Koruda MJ, Behrns KE. Complete fundoplication is not associated with increased dysphagia in patients with abnormal esphageal motility. J Gastrointest Surg 2001; 5: 36-41.

17. Raftopoulos Y, Papasavas P, Landreneau R, Hayetian F, Santucci T, Gagné D. Clinical outcome of laparoscopic antireflux surgery for patients with irritable bowel syndrome. Surg Endosc 2004; 18: 655-9.
18. Zaninotto G, Rizzetto C. Surgical options and outcomes in Barrett's esophagus. Curr Opin Gastroenterol 2007; 23: 452-5.

19. Csendes A, Braghetto I, Burdiles P, Smok G, Henríquez A, Parada F. Regression of intestinal metaplasia to cardiac or fundic mucosa in patients with Barrett's esophagus submitted to vagotomy, partial gastrectomy and duodenal diversion. A prospective study of 78 patients with more than 5 years of follow-up. Surgery 2006; 139: 46-53.

20. Kamolz T, Granderath FA, Pointner R. Does major depression in patients with gastroesophageal reflux disease affect the outcome of laparoscopic antireflux surgery? Surg Endosc 2003; 17: 55-60. 